\title{
Sfrp5 coordinates foregut specification and morphogenesis by antagonizing both canonical and noncanonical Wnt11 signaling
}

\author{
Yan Li, ${ }^{1,3,4}$ Scott A. Rankin, ${ }^{1,3}$ Débora Sinner, ${ }^{1}$ Alan P. Kenny, ${ }^{1}$ Paul A. Krieg, ${ }^{2}$ and \\ Aaron M. Zorn ${ }^{1,5}$ \\ ${ }^{1}$ Cincinnati Children's Research Foundation and Department of Pediatrics, College of Medicine, University of Cincinnati, \\ Cincinnati, Ohio 45229, USA; ${ }^{2}$ Department of Cell Biology and Anatomy, University of Arizona Health Sciences Center, \\ Tuscon, Arizona 85724, USA
}

\begin{abstract}
Cell identity and tissue morphogenesis are tightly orchestrated during organogenesis, but the mechanisms regulating this are poorly understood. We show that interactions between Wnt11 and the secreted Wnt antagonist secreted frizzled-related protein 5 (Sfrp5) coordinate cell fate and morphogenesis during Xenopus foregut development. sfrp5 is expressed in the surface cells of the foregut epithelium, whereas wnt11 is expressed in the underlying deep endoderm. Depletion of Sfrp5 results in reduced foregut gene expression and hypoplastic liver and ventral pancreatic buds. In addition, the ventral foregut cells lose adhesion and fail to form a polarized epithelium. We show that the cell fate and epithelial defects are due to inappropriate Wnt/ $\beta$-catenin and Wnt/PCP signaling, respectively, both mediated by Wnt11. We provide evidence that Sfrp5 locally inhibits Wnt11 to maintain early foregut identity and to allow an epithelium to form over a mass of tissue undergoing Wnt-mediated cell movements. This novel mechanism coordinating canonical and noncanonical Wnt signaling may have broad implications for organogenesis and cancer.
\end{abstract}

[Keywords: Sfrp5; Wnt11; liver; pancreas; foregut; morphogenesis]

Supplemental material is available at http://www.genesdev.org.

Received April 22, 2008; revised version accepted September 4, 2008.

During organogenesis, cell identity and tissue morphogenesis must be coordinated so that epithelial sheets and mesenchymal masses are formed at the correct time and place. The epithelial cells of the digestive system and its associated organs arise from the embryonic endoderm. The endoderm is specified during gastrulation and is initially patterned along its anterior-posterior $(\mathrm{A}-\mathrm{P})$ axis into broad foregut and hindgut domains that can be identified by the localized expression of homeobox genes such as the hhex in the anterior and $c d x$ or vent in the posterior (Grapin-Botton 2005; Moore-Scott et al. 2007). The liver, gall bladder, pancreas, and stomach are then induced from the foregut epithelium by signals from adjacent mesoderm, while intestinal fate is induced in the

\footnotetext{
${ }^{3}$ These authors contributed equally to this work. ${ }^{4}$ Present address: State Key Laboratory of Phytochemistry and Plant Resources, Kunming Institute of Botany, CAS, 132 Lanhei Road, Kunming 650204, China.

${ }^{5}$ Corresponding author.

E-MAIL Aaron.zorn@chmcc.org; FAX (513) 636-4317.

Article is online at http://www.genesdev.org/cgi/doi/10.1101/gad.1687308.
}

posterior endoderm (Zaret 2002; Grapin-Botton 2005). In addition to having distinct developmental potentials, the foregut and hindgut progenitors undergo different morphogenetic movements. For example, the presumptive intestine undergoes extensive elongation, whereas the foregut domain remains a relatively compact diverticulum from which organ buds will emerge (Chalmers and Slack 2000; Horb and Slack 2001; Grapin-Botton 2005). The mechanisms that coordinate the appropriate tissue morphogenesis with endodermal fate are still obscure.

Recent work in Xenopus suggests that differential Wnt signaling is a key event defining foregut versus hindgut progenitors (McLin et al. 2007). Wnt signaling is commonly divided into two main classes. In the "canonical" Wnt/ $\beta$-catenin pathway, ligands interact with a Frizzled $(\mathrm{Fz})$ and LRP6 receptor complex, causing the activation of Dishevelled (Dsh). Dsh inhibits a GSK3 containing degradation complex, resulting in the stabilization and accumulation of nuclear $\beta$-catenin, which interacts with Tcf/Lef transcription factors to activate target gene transcription (Clevers 2006; Macdonald et al. 2007). "Noncanonical" Wnt signaling refers to at least three intra- 
cellular pathways that are independent of $\beta$-catenin (Wallingford and Habas 2005; Semenov et al. 2007). The Wnt/PCP pathway is closely related to the Drosophila planar cell polarity pathway and signals via $\mathrm{Fz}$ and Dsh to activate Rho and Rac GTPases, which signal via Rhoassociated kinase (ROCK) and Jun-N-terminal-kinase (JNK), respectively (Wallingford and Habas 2005). The $\mathrm{Wnt} / \mathrm{Ca}^{2+}$ pathway signals via $\mathrm{Fz}$, G-proteins, and Dsh to mobilize intracellular calcium and activate CamKII (Sheldahl et al. 2003). Finally a Wnt/Ror2 pathway signals via Ror/Ryk receptor tyrosine kinases to activate PI3 kinase, Cdc42, and JNK (Oishi et al. 2003; Schambony and Wedlich 2007). While the details of these noncanonical pathways are unresolved, they all regulate cytoskeleton dynamics and morphogenetic cell behaviors, including epithelial cell polarity, cell adhesion, and cell movement (Wallingford and Habas 2005).

Experiments in Xenopus suggest that during gastrula and early somites stages of development, high $\beta$-catenin activity in the posterior endoderm promotes hindgut fate and represses foregut identity. In contrast, the anterior endoderm experiences low $\beta$-catenin activity, which is necessary and sufficient to maintain foregut identity and to initiate liver and pancreas development (McLin et al. 2007). However the endogenous Wnts involved and the mechanisms that regulate their activity along the A-P axis are unknown.

We postulated that secreted Wnt antagonists are responsible for maintaining low $\beta$-catenin activity in the foregut progenitors. In the gastrula, candidate antagonists include secreted frizzled-related protein 1 (Sfrp1), Sfrp2, Sfrp3 (Frzb1), Sfrp5, Crescent, and Dkk1, all of which are expressed in the anterior mesendoderm (Leyns et al. 1997; Glinka et al. 1998; Pilcher and Krieg 2002; Finley et al. 2003; Kemp et al. 2005). Sfrps sequester Wnt ligands in the extracellular space, preventing them from binding to Frizzled receptors, and thus can inhibit both canonical and noncanonical signaling (Kawano and Kypta 2003). In contrast, Dkk1 specifically inhibits the canonical Wnt pathway by binding to LRP6 (Mao et al. 2001; Semenov et al. 2001). To date, the genetic analysis of Wnt antagonists in the mouse has not revealed a function in foregut development; however, their overlapping expression patterns suggest extensive redundancy (Leimeister et al. 1998; Finley et al. 2003; Kemp et al. 2005). Indeed, mutations in dkk1, sfrp1, sfrp2, or sfrp5, as well as various double and triple mutants, indicate that they have redundant roles in axial trunk formation, but foregut defects have not yet been described in these embryos (Mukhopadhyay et al. 2001; Leaf et al. 2006; Satoh et al. 2006, 2008). In Xenopus, however, sfrp5 is the only Wnt antagonist expressed in the anterior endoderm during early somite stages (Pilcher and Krieg 2002), and thus it is a good candidate for regulating foregut specification during this period.

We show that Sfrp5 is required both for maintaining foregut identity and for correct foregut morphogenesis. Sfrp5-depleted Xenopus embryos have dramatically reduced foregut gene expression and hypoplastic liver and pancreatic buds. In addition, the foregut epithelium does not form properly: The cells lack apical-basal polarity and are loosely adherent, resulting in a collapse of the foregut cavity. We demonstrate that the specification and morphogenesis defects are separable and due to elevated canonical Wnt/ $\beta$-catenin and noncanonical Wnt/ PCP signaling, respectively, both mediated by Wnt11. Our data indicate that Sfrp5 locally inhibits Wnt11 to maintain foregut identity and to allow a foregut epithelial sheet to form over the mass of deep endoderm tissue. This novel mechanism may coordinate cell fate and morphogenesis in many developmental and disease contexts.

\section{Results}

Sfrp5 is expressed in the early foregut epithelium

In Xenopus, Wnt/ $\beta$-catenin activity must be excluded from the anterior endoderm between gastrula and earlysomite stages in order to maintain foregut identity (McLin et al. 2007). To determine if Sfrp5 participates in this process, we examined its expression using in situ hybridization to bisected Xenopus embryos, which exposes the deep endoderm tissue. Sfrp5 transcripts were first detected just after gastrulation in a thin layer of foregut endoderm cells on the archenteron floor (Fig. 1A) - a region that contributes to the liver, ventral pancreas, and stomach (Chalmers and Slack 2000). This foregut-specific expression persists until mid-somite stages (Fig. 1B). Later in development, sfrp5 exhibits a posterior wave of expression, as previously described (Pilcher and Krieg 2002), first in the hepatic endoderm, then the pancreas/ stomach, and finally the proximal small intestine. Thus sfrp5 is expressed at the right time and place to repress Wnt signaling and maintain foregut identity.

\section{Sfrp5 is required for Xenopus gut development}

We tested the function of Sfrp5 by injecting Xenopus laevis embryos with a translation-blocking antisense morpholino oligo (sfrp5-MO). At $3 \mathrm{~d}$ of development, $\sim 80 \%$ of the sfrp5-MO-injected (50 ng) embryos exhibited dramatic gut defects; the guts did not coil or cavitate to form a tube; and the posterior endoderm was frequently expanded (Fig. 1D-G). This effect was dose-dependent, and injection of lower MO doses resulted in a progressively milder phenotype (data not shown). A second morpholino complementary to a different region of the sfrp 5 mRNA resulted in the same phenotype, although less severe, whereas a control oligo with 5 nucleotides mutated (mismatch-MO) had no effect (Fig. 1D; Supplemental Fig. S2). Coinjection of the sfrp5-MO inhibited expression of a synthetic sfrp5:gfp RNA containing the sfrp5 5'-untranslated region (UTR) fused to a GFP coding sequence, while the mismatch-MO did not (Fig. 1H). Finally injection of Xenopus tropicalis embryos with an antisense MO designed to block Xt-sfrp 5 mRNA splicing resulted in a $>90 \%$ reduction of mature sfrp5 transcripts at early somite stage and a gut phenotype 

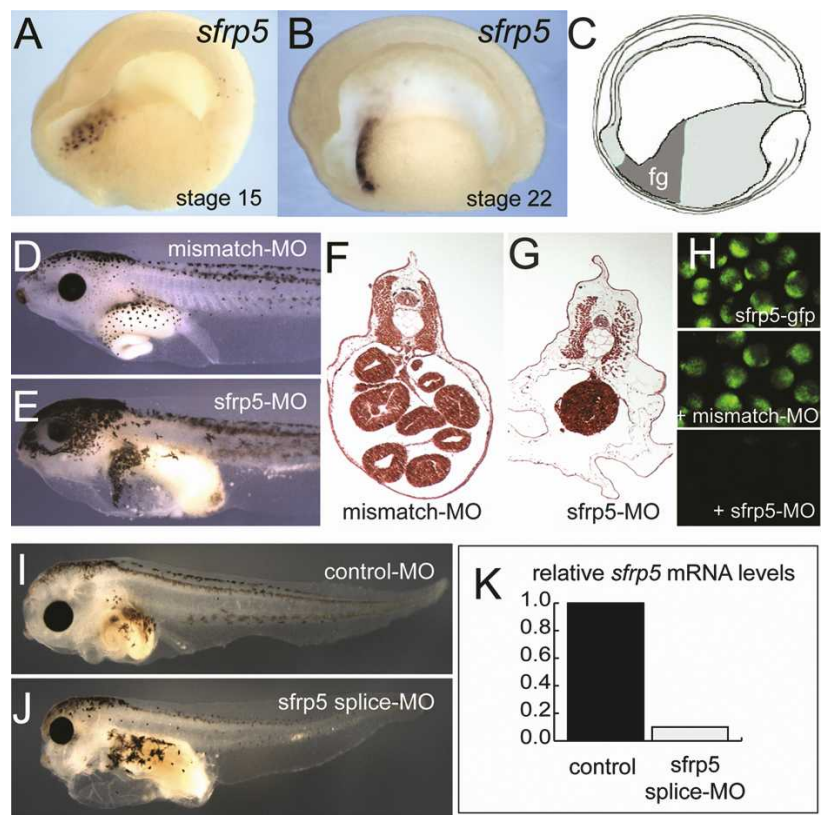

Figure 1. Sfrp5 is required for Xenopus gut development. $(A, B)$ Sfrp5 in situ hybridization to stage 15 (neurula) $(A)$ and stage 22 ( 10 somites) bisected embryos $(B)$ shows expression in the archenteron floor of the ventral foregut. $(C)$ Schematic of a bisected embryo indicating the endoderm (gray) and the ventral foregut $(\mathrm{fg})$. Anterior is left and dorsal is up. $(D)$ Normal intestinal coiling and a translucent hindgut in 3-d-old laevis embryos that were injected with a control mismatch-MO (50 ng). (E) Three-day-old laevis embryos that were injected with a translation-inhibiting sfrp5-MO (50 ng) exhibit a lack of gut coiling and an expanded hindgut $(-80 \%, n>300) .(F, G)$ H\&E-stained transverse section of a mismatch-MO embryo $(F)$ and a sfrp5MO-injected embryo $(G)$ that lacks gut coiling and has no lumen. $(H)$ GFP fluorescence in stage 15 whole embryos injected with a synthetic sfrp5:gfp RNA containing the sfrp5 5'-UTR fused to a GFP coding sequence. Coinjection of the mismatchMO did not inhibit GFP translation, while the sfrp5-MO blocked fluorescence. $(I, I)$ Xenopus tropicalis embryos injected with a control-MO $(I)$ or a sfrp5 splice-MO $(J)$, which causes gut defects similar to the translation blocking sfrp5-MO in laevis $(82 \%, n=50)$. (K) A representative QRT-PCR experiment with primers spanning the Xt. Sfrp5 exon1-exon2 junction shows that the sfrp5 splice-MO reduces mature sfrp5 transcripts by $>90 \%$ in tropicalis embryos. Five embryos were pooled for each sample, and expression was normalized to odc loading control.

indistinguishable from the translation blocking sfrp5$\mathrm{MO}$ (Fig. 1I-K). These data indicate that Sfrp5 function is required for Xenopus gut development.

\section{Sfrp5 is essential for liver and ventral pancreas development}

At tailbud stages, Sfrp5-depleted embryos exhibited smaller foregut cavities that were often filled with disorganized endodermal cells (Fig. 2A,B,D,E). In situ hybridization to isolated gut tubes at stage 42 revealed that sfrp5-MO-injected embryos had hypoplastic liver and ventral pancreatic buds (expressing hhex and $p d x 1$, re- spectively) (Fig. 2G-K). Expression of the liver specification marker for 1 (Fig. $2 \mathrm{M}, \mathrm{N}$ ) and the early foregut genes $h h e x$, foxa2, $h n f 4 \alpha$, and $h n f 1 \beta$ were dramatically reduced or absent in sfrp5-MO embryos (Fig. 2P,Q; Supplemental Fig. S1). This was accompanied by increased expression of the posterior Wnt-target gene vent 2 in the foregut (Fig. $2 \mathrm{~S}, \mathrm{~T}$ ). Expression of the precardiac marker $n k x 2.5$ (stage 20) was unchanged in sfrp5-MO embryos, but the cardiac differentiation marker cardiac-troponin was frequently down-regulated at stage 35 , suggesting a secondary effect on heart formation (Supplemental Fig. S1). Embryos injected with the mismatch-MO were indistinguishable from uninjected controls in all assays (Fig. 2V; Supplemental Fig. S2; Supplemental Table S1). In addition, a synthetic sfrp 5 mRNA lacking the MO target sequence rescued for 1 and hhex expression when it was coinjected into the foregut endoderm with the sfrp5-MO, further confirming the specificity of the knockdown (Fig. 2V; Supplemental Fig. S2; Supplemental Table S1).

Analysis of cell proliferation (phospho-histone $\mathrm{H} 3$ ) and apoptosis (TUNEL) at early somite stages (stage 20), when the foregut defects are first manifested, revealed no significant differences between control and sfrp5-MO embryos (Fig. 2W; data not shown). However later in development (stage 35), we observed a threefold increase in TUNEL-positive cells in the sfrp5-MO foreguts, suggesting that cell death may contribute to the late phenotype (Fig. 2W). Together these data demonstrate that Sfrp5 is required for proper foregut development and endoderm pattering.

\section{Sfrp5 is sufficient to induce foregut identity}

Ectopic expression of sfrp5 mRNA (500 pg) into the posterior-ventral endoderm caused a striking expansion of foregut tissue at the expense of the hindgut (Fig. 2C,F). Embryos exhibited massive liver and ventral pancreatic buds, (Fig. 2I,L), expanded for 1 and hhex expression (Fig. $2 \mathrm{O}, \mathrm{R}$ ), and repression of the posterior marker vent2 (Fig. 2U). This effect was dose-dependent, and injection of lower concentrations of RNA resulted in a progressively milder phenotype (data not shown). At the earlier gastrula stage, hhex expression in the organizer was unchanged in sfrp5 RNA or sfrp5-MO-injected embryos, indicating that the sfrp5-MO had no effect prior to endogenous sfrp5 expression and that sfrp5 RNA injection did not disrupt early axial patterning (Supplemental Fig. S1).

To confirm that Sfrp5 was sufficient to specify foregut identify (rather than just expand the foregut), we isolated posterior endoderm explants from control and sfrp5 RNA-injected embryos at the beginning of gastrulation, prior to morphogenesis. After culturing to stage 15, RTPCR analysis revealed that Sfrp5, like the canonical Wnt antagonists Dkk1 and Gsk3 $\beta$, induced ectopic hhex in the posterior endoderm (Supplemental Fig. S3). These results are consistent with Sfrp5 antagonizing Wnt $/ \beta$ catenin to repress posterior fate and maintain foregut identity. 


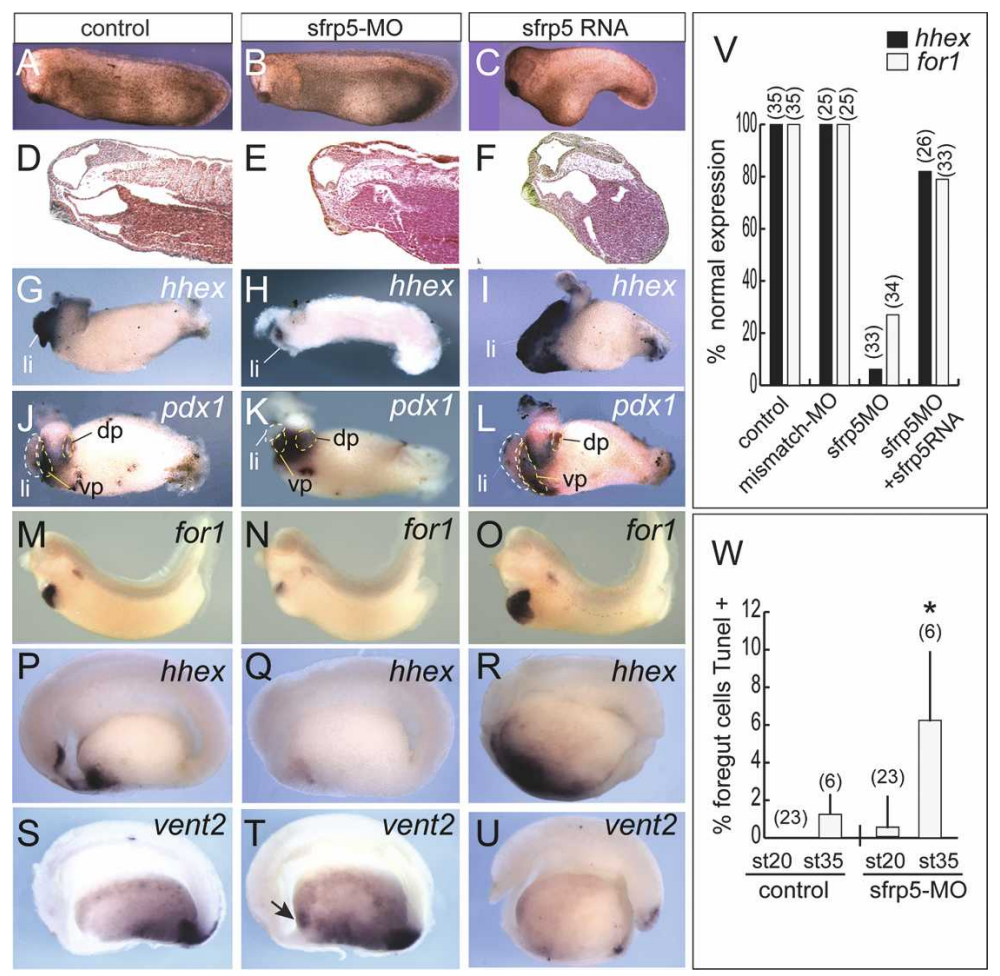

Figure 2. Sfrp5 is required for foregut organogenesis. $(A, D, G, J, M, P, S)$ Control embryos. $(B, E, H, K, N, Q, T)$ Foregut development was impaired by injection of the sfrp5-MO (50 ng). $(C, F, I, L, O, R, U)$ Foregut development was expanded by injection of ectopic sfrp5 RNA $(500 \mathrm{pg})$ into the hindgut. Anterior is to the left and dorsal is up. $(A-C)$ External morphology of stage 30 tailbud embryos. $(D-F)$ Mid-sagittal H\&E-stained sections of stage 30 embryos showing the collapsed foregut cavity in sfrp5-MO embryos (shown in $E$ ). $(G-L)$ In situ hybridization to isolated gut tubes at stage 42 showing that the hhex-expressing liver bud $(G-I)$ and $p d x 1$-expressing ventral pancreatic bud $(J-L)$ were hypoplastic in Sfrp5-depleted embryos and expanded by Sfrp5 overexpression. $(M-O)$ The liver specification marker for 1 was repressed by sfrp 5 depletion and expanded by sfrp5 overexpression at stage $35 .(P-R)$ At stage 20 , the foregut marker hhex was repressed by sfrp5 depletion and expanded by sfrp5 overexpression. $(S-U)$ At stage 20 , the Wnt/ $\beta$-catenin posterior target gene vent 2 was ectopically expressed in the foregut of sfrp5-MO embryos (arrow) and repressed by sfrp5 overexpression. $(V)$ Injection of the mismatch-MO $(50$ ng) had no effect, while coinjection of a synthetic sfrp5 RNA lacking the sequence targeted by the sfrp5MO rescued hhex and for1 expression in sfrp5-depleted embryos. MOs were injected at the eight-cell stage followed by a targeted injection of the sfrp5 RNA (lacking the 5'-UTR; $100 \mathrm{pg}$ ) into the anterior endoderm D1 cells of the 32-cell stage. The histogram shows the percent of embryos with normal expression levels. The number of embryos for each condition is in parentheses, and representative examples are shown in Supplemental Figure S2. (W) Sfrp5-depleted embryos show increased cell death in the foregut at later stages of development, but not at stage 20. The percentage of TUNEL-positive foregut cells is presented \pm standard deviation. The number of embryos counted is in parentheses, with $\sim 75$ foregut cells per embryo at stage 20 and $\sim 150$ foregut cells per embryo at stage $35 .\left(^{\star}\right) P<0.05$ compared with age-matched controls in a Student $t$-test.

\section{Ectopic Sfrp5 repressed hindgut elongation}

Injection of sfrp5 RNA into the posterior endoderm caused a more dramatic foregut expansion than other sfrps or canonical $\mathrm{Wnt} / \beta$-catenin inhibitors such as Dkk1 or Gsk3 $\beta$ (Supplemental Fig. S4B; McLin et al. 2007). This suggested that in addition to promoting foregut fate Sfrp5 might also regulate gut morphogenesis.

In Xenopus the posterior endoderm elongates during tailbud stages as the presumptive intestine begins to lengthen (Larkin and Danilchik 1999; Chalmers and Slack 2000; Horb and Slack 2001). Elongation can be assayed in an explant system where the presumptive hindgut or foregut is dissected at stage 15 , and when cultured until stage 26, the hindgut explants, but not foregut explants, undergo a characteristic elongation (Fig. 3A,F). The molecular mechanisms mediating this are unknown, and we tested whether Wnt signaling was involved and if ectopic Sfrp5 could repress hindgut elongation.

Injection of sfrp5 RNA into the posterior endoderm significantly inhibited elongation of hindgut explants (Fig. 3C,F), but the canonical Wnt antagonist Dkk1 did not (Fig. 3D,F) even though both Sfrp5 and Dkk1 induced ectopic foregut fate (Supplemental Fig. S3). Hindgut elongation was also robustly inhibited by a Disheveled mutant lacking the PDZ domain (dsh- $\triangle P D Z$ ), which specifically disrupts noncanonical Wnt signaling (Fig. 3E,F; Rothbacher et al. 2000; Tada and Smith 2000; Wallingford et al. 2000). These results suggest that noncanonical Wnt signaling is required for hindgut elongation in Xenopus and that Sfrp5 overexpression inhibits this process, resulting in an enlarged foregut (Fig. 2C). Preliminary studies indicate that noncanonical signaling mediates endoderm elongation by promoting polarized cell intercalations similar to those that drive mesodermal convergent-extension (CE) during gastrulation (S.A. Rankin and A.M. Zorn, unpubl.). Interestingly, injection of the sfrp5MO did not enhance foregut elongation, rather the explants were irregularly shaped tended to dissociate (data not shown).

These studies imply that canonical Wnt signaling promotes posterior endoderm fate, whereas noncanonical Wnt signaling regulates gut elongation. The observation that ectopic Sfrp5 can repress both of these activities, along with the sfrp5 loss-of-function phenotype, suggests that Sfrp5 normally restricts both canonical and noncanonical Wnt activities in the foregut in order to maintain foregut identity and prevent inappropriate Wnt-mediated morphogenesis. Consistent with this possibility sfrp5 RNA injection was able to inhibit both canonical and noncanonical Wnt ligands in secondary axis and gastrulation assays (Supplemental Fig. S4). 


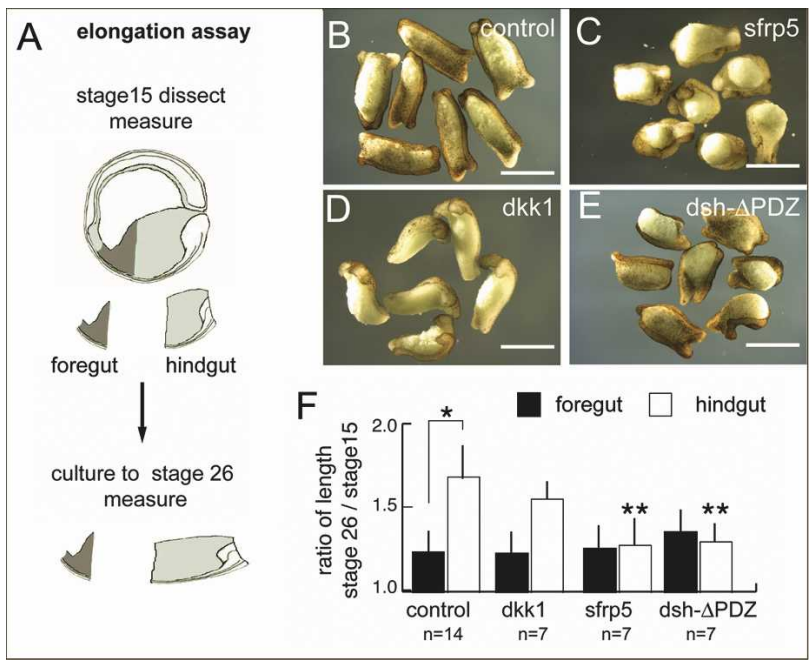

Figure 3. Ectopic Sfrp5 represses hindgut morphogenesis. $(A)$ Hindgut elongation assay. Ventral foregut and hindgut explants were isolated at stage 15 from control embryos and embryos injected with sfrp5, $d k k 1$, or $d s h-\triangle P D Z$ RNA and were cultured until stage 26 . The length of each explant was measured before and after culturing, and the elongation was calculated as a ratio of the lengths at stage 26/stage 15. (B-E) Representative hindgut explants injected with the indicated RNAs. Bars, $0.5 \mathrm{~mm} .(F)$ Sfrp5 and dsh- $\triangle \mathrm{PDZ}$ blocked hindgut elongation, while dkk1 had no effect. The histogram shows the average elongation \pm standard deviation. In pairwise Student $t$-tests, $\left(^{*}\right) P<0.001$, control foregut compared with control hindgut; $\left({ }^{\star \star}\right) P<0.005$ compared with control hindgut.

\section{sfrp5 and wnt11 have complementary expression patterns in the endoderm}

To determine which Wnt ligands Sfrp5 might interact with, we first surveyed all of the known ligands by RTPCR and found that wnt $2 b$, wnt4, wnt5a, wnt5b, wnt $7 b$, wnt 8 , and wnt11 were expressed in the ventral region of the embryo during early somite stages (data not shown). In situ hybridization revealed that wnt $5 a$, wnt $5 b$, wnt8, and wnt 11 were expressed in the posterior mesendoderm and lateral plate mesoderm $(\mathrm{lpm})$ between stages 13 and 20 (Fig. 4A; data not shown), consistent with a role in posterior gut development. This analysis also revealed a previously unappreciated expression domain of wnt11 and its putative receptor $f z 7$ in the deep foregut endoderm underlying the superficial cells that express sfrp5 (Fig. 4A, red arrow). The proximity of their expression domains suggests that Sfrp5 and Wnt11 might interact in vivo.

\section{Sfrp5 binds and antagonizes Wnt5 and Wnt11}

In coinjection experiments with all the candidate ligands, only wnt5a, wnt5b, or wnt11 plasmids rescued the enlarged foregut and shortened hindgut caused by Sfrp5 overexpression (Fig. 4B,C). The fact that Wnt8 had no effect in this assay suggests that while Wnt8 probably signals via the canonical pathway to promote hindgut fate (McLin et al. 2007), it does not regulate hindgut elongation. In support of this, coinjection of a constitutively active JNK plasmid (Liao et al. 2006) rescued posterior elongation while a constitutively active $\beta$-catenin plasmid did not (Fig. 4C).

Although different Sfrps can interact with distinct Wnts (Wang et al. 1997; Galli et al. 2006; Wawrzak et al. 2007), the ligand specificity of Sfrp5 had not been reported. Consistent with our functional data, we found that an epitope-tagged Sfrp5-V5 bound strongly to Wnt5a-HA and Wnt11-HA in coimmunoprecipitations from transfected COS cell lysates (Fig. 4D). In contrast, Sfrp5 exhibited very little binding to Wnt8, in agreement with secondary axis assays where Sfrp5 only partially inhibited Wnt8 activity (Supplemental Fig. S4). Together these data suggest that Wnt11 and/or Wnt5 signal via the noncanonical Wnt pathway to promote gut elongation and that Sfrp5 restricts their activity in the foregut to prevent inappropriate morphogenesis.

\section{Sfrp5 is required to establish a foregut epithelium}

As Wnt signaling can modulate many cell behaviors, we investigated foregut morphogenesis in Sfrp5-depleted
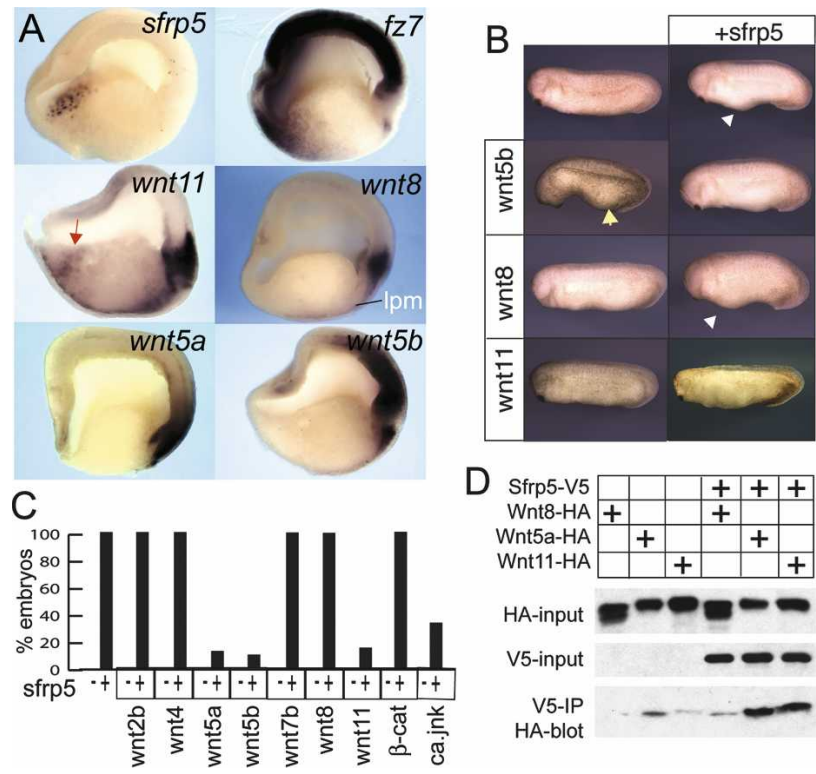

Figure 4. Sfrp5 interacts with Wnt5 and Wnt11. (A) In situ hybridization with the indicated probes to bisected neurula embryos showed that wnt11 and $f z 7$ are expressed in the foregut endoderm (red arrow) underlying the sfrp5 expressing cells. (lpm) Lateral plate mesoderm. (B) Coinjection of $w n t 5 b$ or wnt11 plasmids into the endoderm rescued the enlarged foregut (white arrow) caused by sfrp5 RNA overexpression, while wnt 8 did not. Enlarged hindgut (yellow arrow). (C) Summary of the rescue experiments. Embryos were injected with or without sfrp5 RNA and the indicated wnt plasmid. The histogram shows the percentage of embryos with expanded foreguts. Wnt5a, Wnt5b, Wnt11, and a constitutively active JNK, rescued the Sfrp5 overexpression phenotype, whereas Wnt2b, Wnt4, Wnt7b, Wnt8 and a stabilized $\beta$-catenin did not. $n>15$ embryos for in each condition. $(D)$ Western blotting of a coimmunoprecipitation showing that V5-tagged Sfrp5 preferentially bound to HAtagged Wnt5a and Wnt11 from transfected COS-1 cell extracts. 
embryos in more detail. By removing the anterior neural plate at stage 13, the archenteron floor that expresses sfrp 5 could be examined. The anterior archenteron floor forms the surface of the ventral foregut, and in control embryos, this was a flat sheet of cells, but in Sfrp5-depleted embryos, the cells rounded up and were loosely adherent to one another (Fig. 5A,B). When Sfrp5-depleted embryos were cultured without their neural plates until the tailbud stage, we observed deep endoderm cells erupting through the compromised foregut epithelium (data not shown), apparently being pushed out by the force of hindgut elongation. This explains why the foregut cavity collapses in Sfrp5-deleted embryos (Fig. 2E).

To better understand the cell biological basis of the defect, we examined a range of cell adhesion molecules and cytoskeletal proteins by confocal immunostaining of the foregut. In addition to mediating canonical Wnt signaling, $\beta$-catenin is a component of the cadherin-mediated cell adhesion complex at the plasma membrane that links $\alpha$-catenin and adherens junctions to the actin cytoskeleton. In stage 20 control embryos, the foregut sur- face cells had formed a polarized epithelium; $\beta$-catenin and $\beta 1$-intergin were localized to the basal-lateral cell surfaces; $\alpha$-catenin and f-actin were enriched at the apical cell borders, indicating the presence of adherens junctions; cadherin was enriched at the cell surface; and atypical-PKC (aPKC) was localized to the apical cell surface (Fig. 5 C-E; Supplemental Fig. S5). In contrast, the foregut epithelium was disrupted in Sfrp5-depleted embryos; $\beta$-catenin, aPKC, $\alpha$-catenin, $\beta 1$-integrin, and $\mathrm{f}$-actin were not properly localized (Fig. 5 F-H; Supplemental Fig. S5), indicating a lack of apical-basal polarity and a failure to form adherens junctions. Moreover, the sfrp5MO foregut cells had reduced cadherin at the cell surface, which probably explains the decreased cell adhesion. Western blot and RT-PCR analysis of microdissected foreguts showed that the expression levels of these cytoskeletal components were not substantially reduced in sfrp5-MO-injected embryos (data not shown), suggesting that the loss of Sfrp5 did not affect their transcription or translation, but rather their subcellular localization. The epithelial defect caused by Sfrp5 deple-
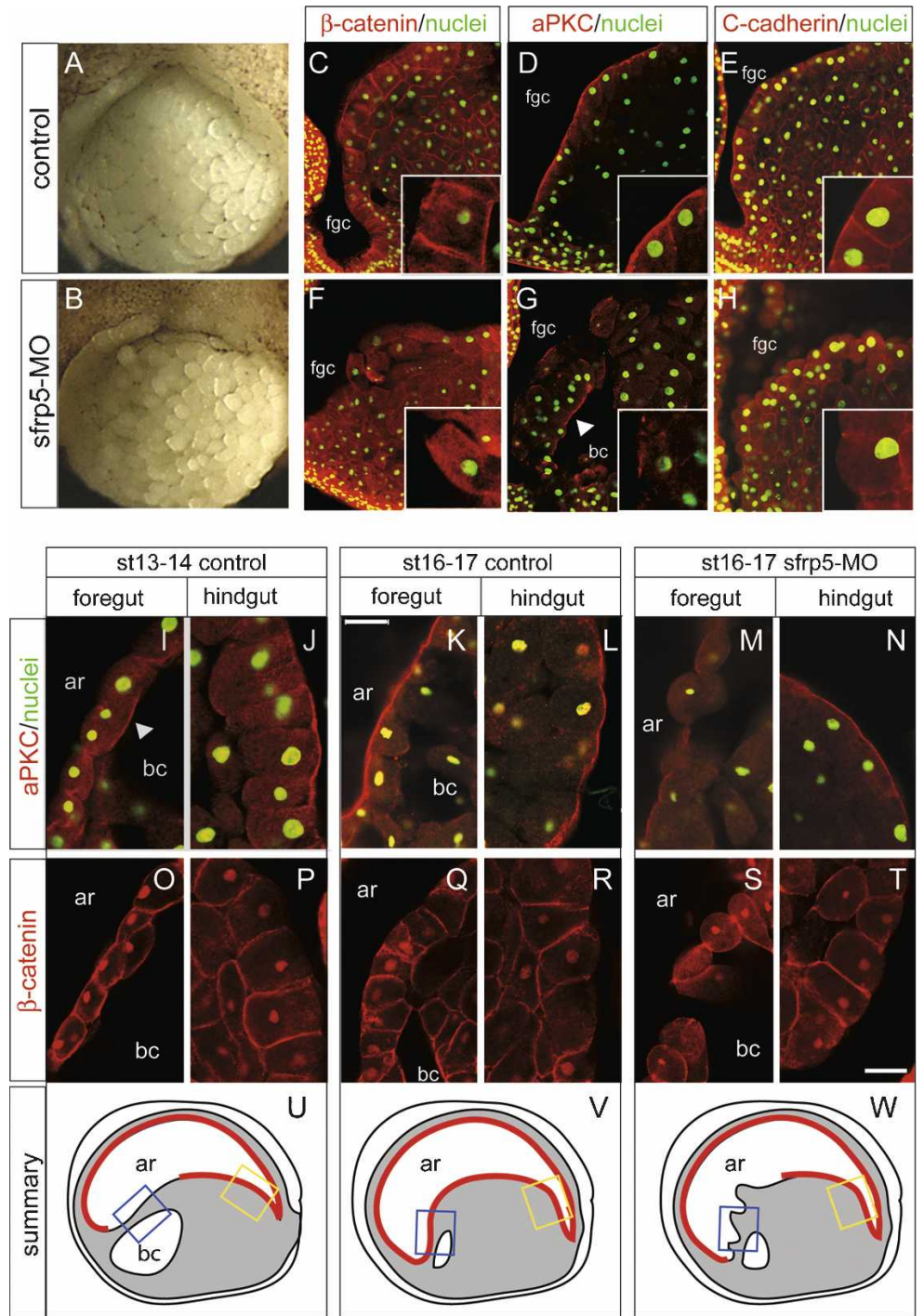

Figure 5. Sfrp5 is required to establish a foregut epithelium. $(A, B)$ Removal of the neural plate at stage 13 reveals the archenteron floor foregut endoderm in control embryos $(A)$ is a flat epithelial sheet of cells, while in sfrp5-MO embryos $(B)$ the cells round up and are loosely adherent. Embryos are oriented anterior down. $(C-H)$ Confocal immunostaining of anti- $\beta$-catenin $(C, F)$, anti-atypical-PKC $(D, G)$, and anti-C-cadherin $(E, H)$ in the foregut region of midsagittally bisected control $(C-E)$ and sfrp5-MOinjected $(F-H)$ embryos at stage 20 (anterior left, dorsal up). Nuclei are counterstained in green. Insets show a high magnification of the foregut surface cells. $(I-T)$ Confocal immunostaining of aPKC $(I-N)$ and $\beta$-catenin $(O-T)$ in the foregut and hindgut surface of control and Sfrp5-depleted embryos (anterior left) at the indicated stages. $(U-W)$ The schematic shows the regions of the foregut (blue box) and hindgut (yellow box) imaged in $I-T$. The red line indicates the regions of the foregut and hindgut surface that exhibit a polarized epithelium with basolateral $\beta$-catenin and apical aPKC. The loss of Sfrp5 results in a failure of the foregut epithelium, reduced cell adhesion and a loss of apical-basal polarity. (fgc) Foregut cavity; (ar) archenteron; (bc) blastocoel. 
tion was rescued by coinjection of sfrp 5 RNA, and the control sfrp5-mismatch-MO did not disrupt the epithelium (Supplemental Fig. S2; Supplemental Table S1).

The disrupted epithelium was only observed in the foregut. The hindgut epithelium and the deep endoderm cells appeared normal. To better understand how the foregut and hindgut epithelium are established and the role that Sfrp5 plays, we examined earlier stages of development. In stages 13-14 control embryos, the anterior archenteron floor, which expresses sfrp5, is a one- to two-cell-layer-thick sheet separating the archenteron from the closing blastocoel cavity. At this stage, $\beta$-catenin was found all around the cell periphery and aPKC was not localized to the apical surface facing the archenteron cavity (Fig. 5I,O,U). Rather some aPKC was localized to the internal surface of the shrinking blastocoel. A few hours later, at stages 16-17 the sfrp5-expressing foregut surface cells had reorganized, forming a polarized epithelium with basolateral $\beta$-catenin and apically localized aPKC (Fig. 5K,Q,V). In contrast to the foregut, the presumptive hindgut epithelium in control embryos was already polarized at stages $13-14$ with basal-lateral $\beta$-catenin and apical aPKC (Fig. 5J,L,P,R). Further analysis indicated that the future hindgut surface cells are a polarized epithelium even prior to gastrulation (data not shown). In Sfrp5-depleted embryos, the hindgut epithelium was unaffected, but the foregut epithelium failed to form. The cells on the anterior archenteron floor remained round with reduced cell contacts, and $\beta$-catenin and aPKC were not properly localized (Fig. 5M,S,W).

This difference between the foregut and hindgut epithelium is consistent with fate mapping studies of the Xenopus gastrula, which show that the foregut epithelium is derived from the endoderm cells of dorsal blastopore lip, which undergo extensive cell rearrangements and shape changes during gastrulation. In contrast, the midgut and hindgut epithelium originates from the vegetal surface of the gastrula, which undergoes very little rearrangement during gastrulation (Keller 1975; Gerhart and Keller 1986; Shook et al. 2004). Our data indicate that Sfrp5 is required to establish the foregut epithelium. In the absence of Sfrp5, the epithelium does not form, the deep endoderm cells erupt into the foregut cavity, and subsequent foregut organ morphogenesis is aberrant.

\section{Wnt/ $\beta$-catenin and Wnt/INK are both up-regulated in Sfrp5-depleted foreguts}

Sfrp5-depleted embryos have two defects-a loss of foregut identity and a disrupted foregut epithelium. It was unclear whether these defects were due to elevated noncanonical Wnt signaling, elevated canonical Wnt activity, or both.

To resolve this, we first sought to determine whether canonical and/or noncanonical signaling was elevated in Sfrp5-depleted foreguts. One measure of active Wnt signaling that has been associated with both noncanonical and canonical pathways is the translocation of Dsh to the plasma membrane (Axelrod et al. 1998; Boutros et al. 1998; Rothbacher et al. 2000; Park et al. 2005; Walling- ford and Habas 2005; Wang et al. 2006; Witzel et al. 2006; Bilic et al. 2007; Zeng et al. 2008). To determine if there was increased membrane localization of Dsh in Sfrp5depleted foreguts, we coinjected a low dose of myctagged Dsh RNA into the anterior endoderm with or without the sfrp5-MO. This low dose of Dsh-myc did not disrupt gastrulation or foregut development. In control embryos, anti-myc immunostaining revealed membrane-localized Dsh in the deep endoderm cells (Fig. 6A; yellow arrow), while surface epithelium had a more diffuse staining. We interpret this to indicate that deep cells normally experience higher levels of canonical Wnt signaling than superficial cells. In contrast, we observed foci of intense membrane Dsh staining in the dissociating foregut surface cells of Sfrp5-depleted embryos (Fig. $6 \mathrm{~A}$; white arrow), suggesting elevated Wnt signaling.

To assay Wnt/ $\beta$-catenin activity, a TOP:flash reporter plasmid was injected with or without the sfrp5-MO. Luciferase assays indicated that endogenous $\beta$-catenin/Tcf transcriptional activity was elevated in the foreguts of Sfrp5-depleted embryos (Fig. 6B). While there is no single transduction component that is universally used by all the noncanonical pathways, JNK activation has been implicated (in at least some contexts) in each of the Wnt/ $\mathrm{PCP}, \mathrm{Wnt} / \mathrm{Ca}^{2+}$, and Wnt/Ror2 branches (Pandur et al. 2002; Yamanaka et al. 2002; Garriock et al. 2005; Schambony and Wedlich 2007). One of the downstream targets of JNK is c-jun, which when phosphorylated is a component of the AP1 the transcription factor complex, and thus JNK activity can be measured using an AP1: luciferase transcriptional reporter (Cheyette et al. 2002; Park and Moon 2002). We found that AP1:luciferase activity was also elevated in sfrp5-MO-injected foreguts (Fig. 6B; Supplemental Fig. S6A), and an in vitro c-jun phosphorylation assay confirmed that JNK activity was increased in Sfrp5-depleted foregut extracts (Fig. 6C). The elevated TOP:flash and AP1:luciferace activity in Sfrp5-depleted foreguts was rescued by coinjection of sfrp5 RNA, while injection of the control mismatchMO had no affect on the reporters (Supplemental Fig. S6A). We conclude that Sfrp5 normally restricts both $\beta$ catenin/Tcf and JNK activity in the Xenopus foregut.

\section{Sfrp5 coordinates foregut specification and morphogenesis by restricting both canonical and noncanonical Wnt signaling, respectively}

We wished to determine whether the increase of either Wnt/ $\beta$-catenin or Wnt/JNK activity could account for the loss of foregut identity or disrupted epithelium in Sfrp5-depleted embryos. To do this, we specifically manipulated either the canonical or noncanonical pathways in the foregut endoderm of control and sfrp5-MO embryos. We assayed the resulting embryos by $\beta$-catenin immunostaining of the epithelium and by hhex and for 1 in situ to determine whether the treatments could mimic or rescue the epithelial defect, the loss of gene expression, or both. Representative results are shown in Figure 6D, and the key data are tabulated in Figure 6E (a complete summary is presented in the Supplemental Table S1). 

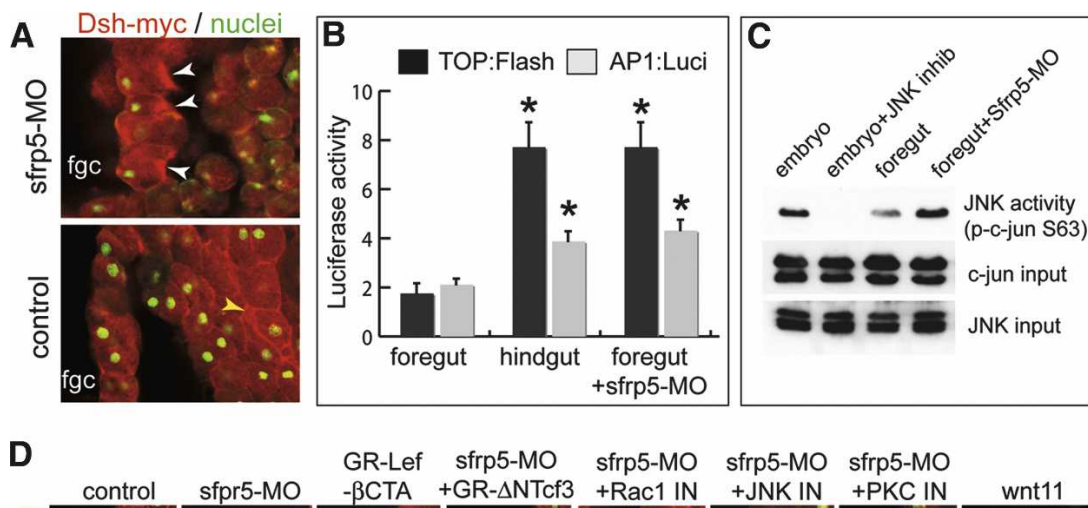

GR-Lef sfrp5-MO
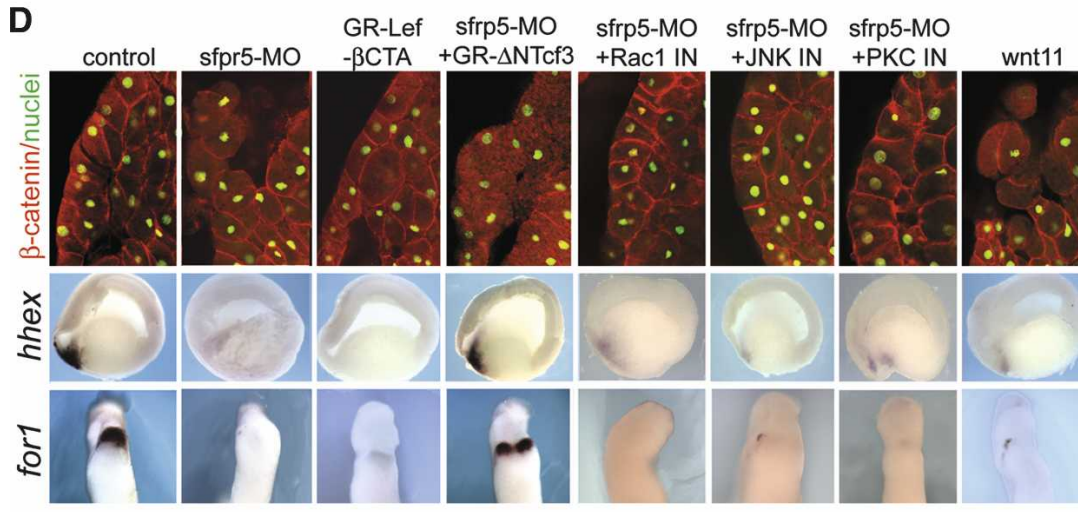

E

intact epithelium $\square$ hhex

for1
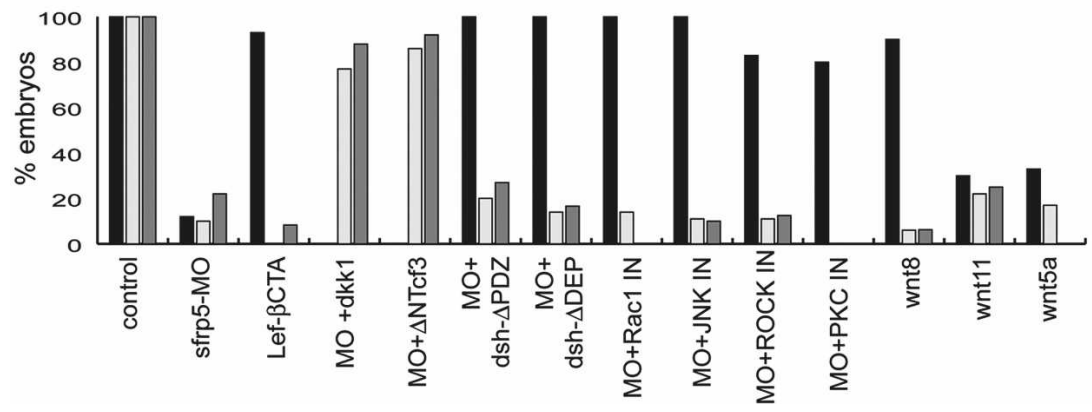

Figure 6. Sfrp5 coordinates foregut morphogenesis and specification by restricting noncanonical Wnt/JNK and canonical Wnt/ $\beta$ catenin, respectively. $(A)$ Membrane-localized Dsh is elevated in Sfrp5-depleted foregut cells. RNA encoding myc-tagged Dsh was injected into the anterior endoderm of control or sfrp5-MO-injected embryos, and its subcellular localization was determined by antimyc immunostaining at stage 18 . In control embryos, membrane-localized Dsh-myc was observed in the deep endoderm cells (yellow arrow) but not in the surface epithelium next to the foregut cavity $(\mathrm{fgc})$. Foci of membranelocalized Dsh-myc were detected in the dissociating surface cells of Sfrp5-depleted foreguts (white arrows). (B) Depletion of sfrp5 results in a specific increase in $\beta$-catenin/Tcf and JNK/AP1 activity in the foregut. TOP: flash or AP1:Luciferase reporter plasmids were injected into either the D1 foregut endoderm cells or the D4 hindgut endoderm cells at the 32-cell stage of control or Sfrp5depleted embryos. The TOP:Flash reporter is an indicator of $\beta$-catenin/Tcf activity, while the AP1:luciferase reporter is an indicator of JNK activation of c-Jun, a component of the AP1 complex. At stage 20, the reporter activity was determined by luciferase assays, in triplicate. The average values normalized to coinjected pRTK:Renila + standard deviation. $\left(^{\star}\right) P<0.05$ in Student $t$-test compared with control foreguts. $(C)$ JNK activity is elevated in Sfrp5-depleted foreguts. Foregut explants were isolated from controls, sfrp5-MO-injected embryos, or embryos treated with a JNK inhibitor (SP600125). The Western blot shows the results of a phospho-c-jun JNK activity assay. (D) Sfrp5 inhibits Wnt/ $\beta$-catenin signaling to maintain foregut gene expression, and inhibits Wnt/PCP signaling to maintain foregut epithelial integrity. The indicated constructs were injected into the D1 foregut endoderm cells at the 32-cell stage. At stage 18, bisected embryos were assayed by anti- $\beta$-catenin immunostaining or by hhex and for 1 in situ at stages 18 and 35, respectively. (E) Representative examples are shown, and a summary is presented in the graph below. A complete summary of all the rescue experiments and controls is presented in Supplemental Table S1.

First, we activated canonical Wnt signaling in the anterior endoderm by targeted injection of a hormone-inducible $\beta$-catenin/Lef fusion protein (GR-LEF- $\beta$ CTA), which activates $\beta$-catenin-target gene transcription in the presence of dexamethasone (dex) (Domingos et al. 2001). Addition of dex to GR-LEF- $\beta$ CTA-injected embryos after gastrulation repressed hhex and for 1 expression but did not disrupt epithelial morphology (Fig. $6 \mathrm{D}, \mathrm{E})$. Moreover coinjection of canonical pathway inhibitors, including an inducible dominant-negative Tcf (GR- $\Delta$ NTcf3) or a Dkk1 plasmid, robustly rescued hhex and for 1 expression in Sfrp5-depleted embryos but could not rescue the epithelial defect (Fig. 6D,E). The rescued for1 liver domain was bifurcated, suggesting that an intact foregut epithelium is required for midline convergence of the organ primordia. These results demonstrate that the loss of foregut identity, but not the disrupted epithelium, is due to elevated Wnt/ $\beta$-catenin signaling in Sfrp5-depleted embryos.

In contrast, epithelial integrity, but not hhex and for 1 expression, was rescued in sfrp5-MO embryos by targeted injection of mutant Dsh constructions (dsh- $\Delta$ PDZ or dsh- $\triangle \mathrm{DEP}$ ) that specifically block noncanonical Wnt signaling (Fig. 6E; Supplemental Fig. 6B; Rothbacher et al. 2000; Tada and Smith 2000; Wallingford et al. 2000). This indicates that the epithelial failure, but not the loss of foregut identity, is due to elevated noncanonical Wnt activity in Sfrp5 deficient embryos. To determine which of the noncanonical pathways caused the epithelial disruption, we inhibited a range of intracellular signaling components. Treating sfrp5-MO embryos after gastrulation with inhibitors of Rac1 (NSC23766), JNK (SP600125), 
ROCK (Y27632), or PKC (BIM or G06976) all rescued the epithelium but did not rescue foregut gene expression (Fig. 6D,E; Supplemental Table S1). Consistent with this, the Rac, JNK, ROCK, and PKC inhibitors had no effect on TOP:flash activity, but as expected, the Rac and JNK inhibitors robustly suppressed the elevated AP1:luciferase reporter activity in sfrp5-depleted embryos (Supplemental Fig. S6C). Inhibitors of CamKII, receptor coupled G-proteins, PI3 kinase, p38, and Cdc42 had little if any effect on the epithelium or gene expression in either sfrp5-MO or control embryos (Supplemental Table S1), suggesting that $\mathrm{Wnt} / \mathrm{Ca}^{2+}$ and Wnt/Ror2 signaling were not involved.

The rescue by Dsh, Rac, JNK, ROCK, and PKC inhibitors suggests that the failure to form a foregut epithelium in sfrp5-MO embryos was due to inappropriate Wnt/PCP signaling in the surface cells. None of the inhibitors tested disrupted the foregut or hindgut epithelial integrity in uninjected control embryos (data not shown), indicating that active Wnt/PCP signaling is not required to form either epithelium. However, several of the PCP pathway inhibitors did affect other aspects of gut morphogenesis. The JNK inhibitor repressed hindgut elongation, and the embryos appeared similar to those injected with sfrp5 RNA (data not shown). The ROCK and PKC inhibitors did not block gut elongation but rather resulted in bifurcated liver and heart domains, indicating a failure of midline convergence, while treatment with the Rac inhibitor blocked both convergence and extension (Supplemental Table S1). Together with the results from Figure 3, these data suggest that Wnt/ PCP signaling promotes the convergence and extension of the deep endoderm and that Sfrp5 must repress this activity in the foregut surface to form an epithelium. In summary, both canonical and noncanonical Wnt signaling are elevated in Sfrp5-depleted foreguts, resulting in the loss of foregut gene expression and a failure of the foregut epithelium.

\section{Wnt11 overexpression phenocopies loss of Sfrp5}

Our results suggest that the elevated $\beta$-catenin and PCP activity in Sfrp5-depleted embryos was due to a local increase in of Wnt11, Wnt5, and/or Wnt8 concentration. To test this, we asked if overexpression of these ligands in the foregut endoderm could phenocopy the sfrp5-MO.

Targeted injection of a wnt8 plasmid into presumptive anterior endoderm inhibited hhex and for 1 expression but did not disrupt the epithelium (Fig. 6E). In contrast, targeted overexpression of Wnt11 or Wnt5 plasmids inhibited both foregut gene expression as well as epithelium formation (Fig. 6D,E), suggesting that Wnt11 and Wnt5 can signal via both the canonical and noncanonical pathways in the foregut. This was surprising because although Wnt11 and Wnt5 have been shown to stimulate either canonical or noncanonical signaling depending on the cellular context (Heisenberg et al. 2000; Tada and Smith 2000; Pandur et al. 2002; Garriock et al. 2005; Tao et al. 2005), it was previously unclear whether they could stimulate both pathways in the same cells. Indeed, the canonical and noncanonical Wnt pathways are thought to inhibit one another (Kuhl et al. 2001; Topol et al. 2003; Mikels and Nusse 2006; Caneparo et al. 2007).

\section{Sfrp5 antagonizes endogenous Wnt11 signaling}

To test whether endogenous Sfrp5-Wnt11 interactions coordinate endoderm cell fate and morphogenesis, we injected embryos with an antisense wnt11 morpholino oligo (wnt11-MO). In control experiments, injection of the wnt1-MO into the dorsal mesoderm resulted in gastrulation and heart defects, as previously described (Pandur et al. 2002; Schambony and Wedlich 2007) (Supplemental Fig. S7). However, when we targeted the wnt11$\mathrm{MO}$ injection to the presumptive anterior endoderm, most of the embryos gastrulated normally, but at stage 20 we observed expanded hhex expression and reduced levels of the posterior marker vent2 (Fig. 7), which was accompanied by a modest decrease in TOP:Flash activity in the hindgut (Supplemental Fig. S8). As a control, we assayed some of the wnt11-MO embryos at the gastrula stage and found no changes in TOP:Flash activity or hhex expression in the organizer, confirming that the earlier role of maternal Wnt11 in axis specification was unaffected in these experiments (Supplemental Figs. S7, S8). Thus reduced Wnt11 levels in the endoderm resulted in decreased $\beta$-catenin signaling and expanded foregut identity.

The foregut epithelium was intact in Wnt11-depleted embryos, as judged by $\beta$-catenin and aPKC immunostaining, but the shape of the surface cells was irregular (Fig. 7) and the foregut cavity was abnormally narrow similar to sfrp5 RNA overexpression (data not shown). The AP1:luciferase reporter activity was also reduced in the Wnt11-depleted endoderm (Supplemental Fig. S8), suggesting that endogenous JNK activity is regulated by Wnt11, consistent with previous reports (Pandur et al. 2002; Garriock et al. 2005). Later in development, the liver and heart were bifurcated (Fig. 7; Supplemental Fig. S7), similar to embryos treated with the Rac, ROCK, or PKC inhibitors (Supplemental Table S1), indicating that Wnt11/PCP signaling is required for the midline convergence of the foregut organs (Garriock et al. 2005).

Finally we tested whether reducing Wnt11 levels was sufficient to rescue foregut development in Sfrp5-depleted embryos. Coinjecting wnt11-MO along with the sfrp5-MO into anterior endoderm rescued both the Wnt/ PCP-dependent epithelial defect and the Wnt/ $\beta$-cateninregulated foregut gene expression. In addition, coinjection of the wnt11-MO reduced the elevated TOP:Flash and AP1:reporter activity in the foreguts of Sfrp5-depleted embryos (Supplemental Fig. S8). These data demonstrate that Sfrp5 antagonizes Wnt11 activity in the foregut, thereby restricting both canonical Wnt and noncanonical Wnt signaling to coordinate foregut fate and morphogenesis (Fig. 7B).

\section{Discussion}

Sfrp5 coordinates foregut specification and morphogenesis

Previous studies have predicted a model where differential Wnt/ $\beta$-catenin signaling controls endodermal cell 
Figure 7. Sfrp5 antagonizes endogenous Wnt11 signaling. (A) Reducing endogenous Wnt11 rescues Sfrp5-depleted embryos. Embryos were injected in the D1 anterior endoderm at the 32-cell stage with a wnt11-MO, sfrp5-MO, or both the wnt11-MO and the sfrp5-MO. At stage 18, the foregut epithelium was assayed by anti- $\beta$-catenin or aPKC immunostaining of bisected embryos and gene expression was examined by in situ hybridization. Injection of the wnt11-MO expanded hhex and reduced vent2 expression. Coinjection of the wnt11-MO and sfrp5MO rescued both the epithelial integrity and the changes in gene expression observed with sfrp5-MO alone. (fgc) Foregut cavity. (B) A model of how Sfrp5 restricts both Wnt $11 / \beta$-catenin and Wnt11/PCP activity to coordinate foregut specification and morphogenesis.

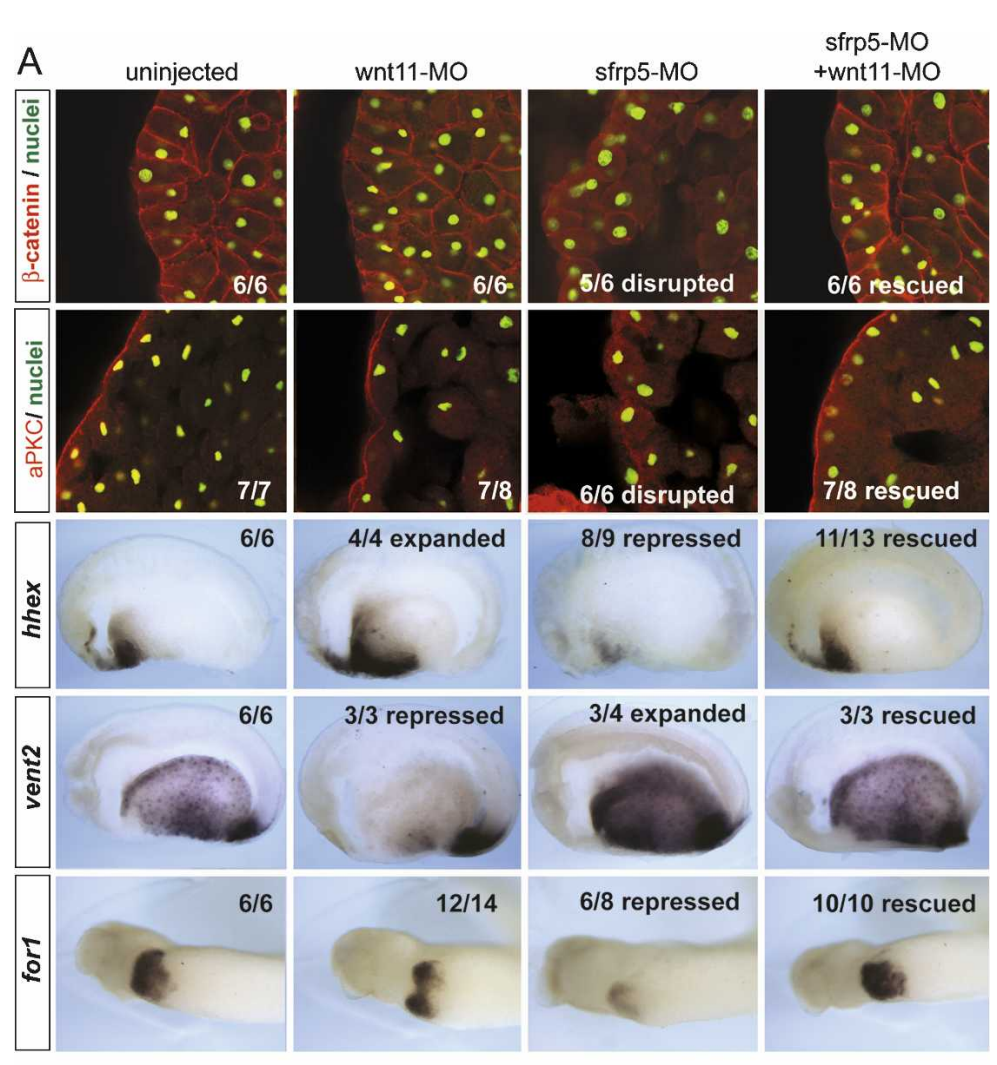

B

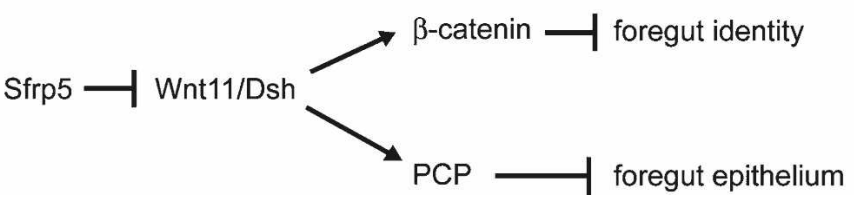

fate along the A-P axis. In this model, Wnt ligands stimulate high $\beta$-catenin activity in the posterior endoderm to promote vent2 expression and intestinal fate. Vent2 in turn represses hhex and foxa2, which are essential for foregut organogenesis (McLin et al. 2007). The model predicts that secreted Wnt antagonists in the anterior endoderm protect foregut progenitors and repress Wnt/ $\beta$-catenin signaling. Until now, the endogenous antagonists and ligands involved in this process were unknown. We show that depletion of Sfrp5 results in loss of foregut gene expression, as predicted by the model. Surprisingly, the loss of Sfrp5 also prevents formation of the foregut epithelium, which results in a collapse of the foregut cavity and disrupted organ bud morphogenesis. We show that the defects in cell identity and epithelial integrity are separable and are due to inappropriate Wnt/ $\beta$-catenin and Wnt/PCP signaling, respectively. Our data suggest that Wnt11 activates both these pathways in the endoderm and that Sfrp5 locally inhibits both canonical and noncanonical Wnt11 signaling to coordinate foregut fate and morphogenesis (Fig. 7B).
Noncanonical Wnt/PCP signaling regulates gut morphogenesis.

Between stages 15-30, the Xenopus gut elongates along the A-P axis and bilateral organ primordia converge at the ventral midline. Our results indicate that this is mediated by Wnt/PCP signaling, consistent with reports that redundant Wnt11, Wnt11-R, and Wnt4a ligands promote midline CE of endodermal progenitors in zebrafish (Matsui et al. 2005). While future studies will be needed to precisely describe the cell behaviors mediating CE of the Xenopus gut and to determine how these are regulated by the PCP pathway, our inhibitor studies shed some light on this. Our results suggest that Dsh (PDZ domain) and JNK activity are required for gut elongation, whereas ROCK and PKC activity are required for midline convergence of the foregut organs, with Dsh (DEP domain) and Rac activity being essential for both convergence and extension. This is reminiscent of CE in the gastrula mesoderm where Wnt11 signals via Dsh (both the PDZ and DEP domains), Rac, JNK, Rho, ROCK, and PKC to coordinate dynamic cell behaviors, with each of 
the components having both distinct and overlapping functions (Yamanaka et al. 2002; Habas et al. 2003; Kinoshita et al. 2003; Tahinci and Symes 2003; Kim and Han 2005).

\section{Sfrp5 locally represses Wnt11/PCP signaling to establish a foregut epithelium}

In addition to mediating CE movements, classic PCP signaling regulates cell polarity within an epithelium. For example, Wnt5a- and Sfrp3-mediated PCP signaling controls proximal-distal polarity of sensory cells in the mouse inner ear epithelium (Qian et al. 2007). The PCP pathway also regulates apical-basal cell polarity in the Xenopus ectoderm (Dollar et al. 2005). In contrast to these examples where PCP signaling is essential to maintain a polarized epithelium, we show that Sfrp5 is required to repress PCP activity in order to establish a polarized foregut epithelium.

In Xenopus, the ventral endoderm is not a thin sheet as in mice and chicks but rather a mass of tissue. During Xenopus development, the foregut epithelium must form from anterior endoderm that underwent significant cell rearrangements during gastrulation; moreover, this ventral foregut epithelium must form over a mass of deep endoderm that continues to undergo Wnt/PCP-mediated cell movements. In contrast, the surface of the hindgut is already a polarized epithelium prior to gastrulation (Keller 1975; Gerhart and Keller 1986; Shook et al. 2004) and appears to be unaffected by perturbations in Wnt or Sfrp5 activity. In Sfrp5-depleted embryos, the cells of the archenteron floor become loosely adherent and do not establish a proper epithelium with basal-lateral-enriched $\beta$-catenin and apical aPKC. Our data suggest that Sfrp5 acts locally, at the surface of the endodermal mass, to inhibit inappropriate Wnt11-mediated PCP signaling and allow the formation of the ventral foregut epithelium. Interestingly, in Sfrp5-depleted embryos, the PCP-dependent defect was more localized than the Wnt/ $\beta$-catenin-dependent gene expression defects. Cell dissociation and the loss of cadherins and $\beta 1$-integrins from the membrane were limited to the sfrp 5 expressing cells and their immediate neighbors, whereas the decreased $h$ hex expression was detected up to $10-15$ cells from the Sfrp5 source. Interestingly hhex ${ }^{-/-}$mutant mouse embryos also exhibit foregut epithelium defects and cell shedding (Bort et al. 2006), suggesting that Hhex, Sfrp5, and Wnt11 may function in an autoregulatory loop.

Wnt11 signaling regulates a diversity of cell behaviors during gastrulation. Based on these, we can postulate a number of mechanisms where Sfrp5 could promote epithelium formation by locally restricting Wnt11 activity. First, Sfrp5 might stop the surface cells from undergoing the PCP-mediated cell movements that the deep cells experience, which is probably a prerequisite to forming an epithelium. This interpretation is consistent with our inhibitor experiments where blocking any one of the Rac, ROCK, JNK, or PKC effectors of the PCP pathway is sufficient to rescue the epithelial defect in Sfrp5-de- pleted embryos. This is similar to gastrulation where inhibition of Rac, Rho, or PKC activity is sufficient to inhibit CE movements. Wnt11 signaling has also been implicated in modulating E-cadherin-mediated cell cohesion, by regulating the dynamics of Rab5-mediated cadherin endocytosis and recycling to the membrane (Ulrich et al. 2005; Witzel et al. 2006; Ogata et al. 2007). Sfrp5 might locally repress this activity and slow down cadherin cycling in the surface cells, leading to enhanced cell cohesion and allowing the formation of adherens junctions. Wnt11/Fz7 can also signal through PKC to promote tissue separation behavior (Winklbauer et al. 2001), and therefore, Sfrp5 might increase adhesion between the surface cells and the deep endoderm as the blastocoel closes. It is also possible that Sfrp5 does not completely inhibit Wnt11 signaling but rather restricts it to generate a local concentration gradient of Wnt activity across the surface cells. In this case, Sfrp5 might restrict Wnt11-induced Fz accumulation to the future basal side of the surface cells, which may increase persistent cell contacts on that surface (Witzel et al. 2006) and thereby initiate an apical-basal bias. It is also possible that Sfrp5 acts indirectly by repressing the transcription of some Wnt11/JNK/AP1 target gene /although this seams unlikely since repression of ROCK or PKC rescued the sfrp5-MO phenotype but did not reduce AP1 activity). Finally, it is possible that a number of these mechanisms cooperate to establish the foregut epithelium.

\section{Wnt11 signals regulate both the canonical and noncanonical pathways}

Depending on the context, Wnt11 has been shown to stimulate either noncanonical or canonical signaling. In Xenopus, for example, maternal Wnt11 interacts with Fz7/LRP6 to activate $\beta$-catenin during dorsal axis specification (Tao et al. 2005). Then at gastrula stages, zygotic Wnt1 1 in the chordomesoderm signals via the PCP pathway to regulate convergent extension (Heisenberg et al. 2000; Tada and Smith 2000). Later still, Wnt11/JNK-mediated noncanonical signaling promotes cardiac development (Pandur et al. 2002; Garriock et al. 2005). However, it was previously unclear whether both pathways could be active in the same tissue; indeed, these two branches are commonly thought to antagonize one another (Kuhl et al. 2001; Topol et al. 2003; Mikels and Nusse 2006; Caneparo et al. 2007). Our results suggest that Wnt11 signals via both the canonical and noncanonical pathways in the endoderm.

\section{Wnt11 and Sfrp5 in heart development}

The development of the heart and the foregut organs are linked through a series of reciprocal signaling interactions, several of which involve Wnt signaling. Wnt antagonists in the anterior endoderm are thought to induce cardiac fate in the adjacent mesoderm by repressing $\beta$ catenin activity (Tzahor and Lassar 2001; Foley and Mercola 2005). Then later in development, the migration of 
cardiac tissue to the midline is regulated by the foregut endoderm and Wnt11/JNK/PKC signaling (Pandur et al. 2002; Garriock et al. 2005). It is likely that Sfrp5 regulates some of these events. For example, cardiac troponin expression was often repressed in Sfrp5-depleted embryos perhaps due to elevated $\beta$-catenin activity. In addition, depletion of both Sfrp5 and Wnt11 rescued the bifurcated heart observed in embryos injected with the Wnt11-MO alone, suggesting that the balance of Sfrp5 and Wnt11 may also regulate the proper midline convergence of foregut organs.

\section{Sfrps in cancer and metaplasia}

Our results may also have implications for homeostasis of adult gut epithelium and cancer. In humans, downregulation of Sfrp1, Sfrp2, Sfrp4, and Sfrp5 has been correlated with Barrett's esophagus (Zou et al. 2005) a precancerous neoplasia where the esophageal epithelium is transformed into more posterior intestinal fates. Moreover, Sfrps are known to be tumor suppressors that are epigenetically inactivated in many cancers as they progress to aggressive carcinomas (Nojima et al. 2007; Nomoto et al. 2007). Our data suggest that loss of Sfrp function might result in both elevated $\beta$-catenin-mediated cell proliferation as well as inappropriate noncanonical Wnt signaling, which may contribute to the loss of epithelial integrity associated with metastasis.

\section{Materials and methods}

\section{Embryo manipulations and microinjections}

Embryo manipulation, explant culture, and microinjections were performed as described previously (McLin et al. 2007). In some cases, such as for Wnt ligands, we injected plasmids that express after the blastula stage to avoid interfering with early axis specification or gastrulation. Unless otherwise indicated, the following amounts of morpholino oligos, RNAs, and plasmids were injected. The following morpholino oligos were used: $50 \mathrm{ng} X$. laevis translation-inhibiting sfrp5-MO $\left(5^{\prime}\right.$-CTCCCAAATGTG GAAAAAGCCAATG-3'), mismatch-MO (5'-CTCCgAAATcT GcAAAAAGgCAtTG-3'), Translation-inhibiting sfrp5-MO2 (5'CAAAATATTCCACTGAAATCGCATG-3'); $25 \mathrm{ng} X$. tropicalis sfrp5 splice-inhibiting MO (5'-AAGGAAGGAAGTATTTTAC CCTGGTG-3'), Wnt11-MO (Pandur et al. 2002). The following RNAs were used: $800 \mathrm{pg}$ of GR-Lef- $\beta$ CTA and GR- $\Delta$ NTcf3 (McLin et al. 2007); 500 pg of pT7TS-Sfrp5, pCS107-Dkk1, pCS2-Sfrp2, pCS107-Crescent, pCS2 + Gsk3 $\beta$; 300 pg of pCS2Dsh-myc (Sokol et al. 1995), pCS2-Dsh- $\triangle \mathrm{PDZ}(\mathrm{D} 2)$, and pCS2Dsh- $\triangle \mathrm{DEP}(\mathrm{D} 6)$ (Rothbächer et al. 2000); 100 pg of pCS2 + sfrp5-UTR-GFP. The following plasmids were used: $250 \mathrm{pg}$ of pCS2 + Xwnt8, pCS2 + Xwnt11, pCS2 + Xwnt2b, pCS2 + mwnt4, pCS107-Xwnt5b, pCS2 + Xwnt7b, pCS 2 + pt- $\beta$-catenin, pCS2 + c.a.JNK (Liao et al. 2006). Dex (1 $\mu$; for GR constructs) and the following cell-soluble inhibitors were dissolved in DMSO and added to the media at stages 11-12; JNK inhibitor SP600125 (50-100 $\mu \mathrm{M})$, Rac1 inhibitor NSC23766 (100-200 $\mu M)$, Cdc42 inhibitor casin $(50 \mu \mathrm{M})$, PKC inhibitor BIM $(40 \mu \mathrm{M})$, $\mathrm{Ca}^{2+}$-dependant PKC inhibitor Go6976 $(40 \mu \mathrm{M})$, G-protein inhibitor pertussis toxin (200-300 ng/mL), and CamKII inhibitor, KN-93 $(20 \mu \mathrm{M})$.

\section{In situ hybridization, immunohistochemistry, and PCR}

In situ hybridization, RT-PCR, and immunohistochemistry analyses were performed as previously described (Sinner et al. 2004; McLin et al. 2007). The following primary antibodies were used: rabbit anti- $\beta$-catenin (1:250; H-102, Santa Cruz Biotechnologies), mouse anti-C-cadherin (1:250; 6B6, Developmental Studies Hybridoma Bank [DSHB]), mouse anti-E-cadherin $(1: 250 ; 5 \mathrm{D} 3, \mathrm{DSHB})$, mouse anti- $\beta 1$-integrin $(1: 500,8 \mathrm{C} 8, \mathrm{DSHB})$, rabbit anti-atypical-PKC (1:100; sc-216 Santa Cruz Biotechnologies), mouse anti-myc (1:250; 9E10, DSHB). The following secondary antibodies were used: goat anti-rabbit-cy5 (1:300; Jackson Immunoresearch) and goat anti-mouse-cy5 (1:500; Jackson Immunoresearch). Nuclei were counterstained with Sytoxgreen.

Coimmunoprecipitations, TOP:Flash, AP1:Luciferase, and JNK assays

For coimmunoprecipitations, $3 \mu \mathrm{g}$ of the following plasmidspcDNA6-V5, pcDNA6-sfrp5-V5, pCS107Xwnt8-HA, pCS2 + Xwnt11-HA (Tao et al. 2005), or pC107 + Xwnt5a-HA-were transfected into COS-1 cells using FuGene (Roche). After $36 \mathrm{~h}$, cleared cell lysates were mixed and immunoprecipicated with anti-V5 antibodies ( $1 \mu \mathrm{g}$; Invitrogen) as previously described (Sinner et al. 2004). Standard Western blotting with rat anti-HA (1:500; 3F10, Roche) and anti-V5-HRP antibodies were used to detect the precipitated tagged proteins. Top-flash (150 pg), AP1:luciferase (150 pg), and pRL-TK renilla (25 pg) plasmids were injected into embryos as indicated in the text. Embryo extracts were prepared (five embryos per sample), and luciferase activity was measured using a commercial kit (Promega). JNK activity was assayed using an in vitro phosphorylation kit (Cell Signaling Technologies).

\section{Acknowledgments}

We thank Drs. J. Heasman, A. Kuan, R. Moon, S. Sokol, J. Wanningford, and Y. Zheng for reagents. We are grateful to Janet Heasman, Matt Kofron, Sumeda Nandadasa, Yi Zheng, Gail Deutsch, Jim Wells, and members of the Wells and Zorn laboratories for helpful comments on this work. This study was supported by NIH grants HD42572 and DK70858 to A.M.Z.

\section{References}

Axelrod, J.D., Miller, J.R., Shulman, J.M., Moon, R.T., and Perrimon, N. 1998. Differential recruitment of Dishevelled provides signaling specificity in the planar cell polarity and Wingless signaling pathways. Genes \& Dev. 12: 2610-2622.

Bilic, J., Huang, Y.L., Davidson, G., Zimmermann, T., Cruciat, C.M., Bienz, M., and Niehrs, C. 2007. Wnt induces LRP6 signalosomes and promotes dishevelled-dependent LRP6 phosphorylation. Science 316: 1619-1622.

Bort, R., Signore, M., Tremblay, K., Barbera, J.P., and Zaret, K.S. 2006. Hex homeobox gene controls the transition of the endoderm to a pseudostratified, cell emergent epithelium for liver bud development. Dev. Biol. 290: 44-56.

Boutros, M., Paricio, N., Strutt, D.I., and Mlodzik, M. 1998. Dishevelled activates JNK and discriminates between JNK pathways in planar polarity and wingless signaling. Cell 94: 109-118.

Caneparo, L., Huang, Y.L., Staudt, N., Tada, M., Ahrendt, R., Kazanskaya, O., Niehrs, C., and Houart, C. 2007. Dickkopf-1 regulates gastrulation movements by coordinated modulation of Wnt $/ \beta$ catenin and Wnt/PCP activities, through in- 
teraction with the Dally-like homolog Knypek. Genes \& Dev. 21: 465-480.

Chalmers, A.D. and Slack, J.M. 2000. The Xenopus tadpole gut: Fate maps and morphogenetic movements. Development 127: 381-392.

Cheyette, B.N., Waxman, J.S., Miller, J.R., Takemaru, K., Sheldahl, L.C., Khlebtsova, N., Fox, E.P., Earnest, T., and Moon, R.T. 2002. Dapper, a Dishevelled-associated antagonist of $\beta$-catenin and JNK signaling, is required for notochord formation. Dev. Cell 2: 449-461.

Clevers, H. 2006. Wnt $/ \beta$-catenin signaling in development and disease. Cell 127: 469-480.

Dollar, G.L., Weber, U., Mlodzik, M., and Sokol, S.Y. 2005. Regulation of Lethal giant larvae by Dishevelled. $\mathrm{Na}$ ture 437: 1376-1380.

Domingos, P.M., Itasaki, N., Jones, C.M., Mercurio, S., Sargent, M.G., Smith, J.C., and Krumlauf, R. 2001. The Wnt/ $\beta$ catenin pathway posteriorizes neural tissue in Xenopus by an indirect mechanism requiring FGF signalling. Dev. Biol. 239: $148-160$.

Finley, K.R., Tennessen, J., and Shawlot, W. 2003. The mouse secreted frizzled-related protein 5 gene is expressed in the anterior visceral endoderm and foregut endoderm during early post-implantation development. Gene Expr. Patterns 3: $681-684$.

Foley, A.C. and Mercola, M. 2005. Heart induction by Wnt antagonists depends on the homeodomain transcription factor Hex. Genes \& Dev. 19: 387-396.

Galli, L.M., Barnes, T., Cheng, T., Acosta, L., Anglade, A., Willert, K., Nusse, R., and Burrus, L.W. 2006. Differential inhibition of Wnt-3a by Sfrp-1, Sfrp-2, and Sfrp-3. Dev. Dyn. 235: 681-690.

Garriock, R.J., D'Agostino, S.L., Pilcher, K.C., and Krieg, P.A. 2005. Wnt11-R, a protein closely related to mammalian Wnt11, is required for heart morphogenesis in Xenopus. Dev. Biol. 279: 179-192.

Gerhart, J. and Keller, R. 1986. Region-specific cell activities in amphibian gastrulation. Annu. Rev. Cell Biol. 2: 201-229.

Glinka, A., Wu, W., Delius, H., Monaghan, A.P., Blumenstock, C., and Niehrs, C. 1998. Dickkopf-1 is a member of a new family of secreted proteins and functions in head induction. Nature 391: 357-362.

Grapin-Botton, A. 2005. Antero-posterior patterning of the vertebrate digestive tract: 40 years after Nicole Le Douarin's Ph.D. thesis. Int. J. Dev. Biol. 49: 335-347.

Habas, R., Dawid, I.B., and He, X. 2003. Coactivation of Rac and Rho by Wnt/Frizzled signaling is required for vertebrate gastrulation. Genes \& Dev. 17: 295-309.

Heisenberg, C.P., Tada, M., Rauch, G.J., Saude, L., Concha, M.L., Geisler, R., Stemple, D.L., Smith, J.C., and Wilson, S.W. 2000. Silberblick/Wnt11 mediates convergent extension movements during zebrafish gastrulation. Nature 405: 76-81.

Horb, M.E. and Slack, J.M. 2001. Endoderm specification and differentiation in Xenopus embryos. Dev. Biol. 236: 330343.

Kawano, Y. and Kypta, R. 2003. Secreted antagonists of the Wnt signalling pathway. J. Cell Sci. 116: 2627-2634.

Keller, R.E. 1975. Vital dye mapping of the gastrula and neurula of Xenopus laevis. I. Prospective areas and morphogenetic movements of the superficial layer. Dev. Biol. 42: 222-241.

Kemp, C., Willems, E., Abdo, S., Lambiv, L., and Leyns, L. 2005. Expression of all Wnt genes and their secreted antagonists during mouse blastocyst and postimplantation development. Dev. Dyn. 233: 1064-1075.
Kim, G.H. and Han, J.K. 2005. JNK and ROK $\alpha$ function in the noncanonical Wnt/RhoA signaling pathway to regulate Xenopus convergent extension movements. Dev. Dyn. 232: 958-968.

Kinoshita, N., Iioka, H., Miyakoshi, A., and Ueno, N. 2003. PKC $\delta$ is essential for Dishevelled function in a noncanonical Wnt pathway that regulates Xenopus convergent extension movements. Genes \& Dev. 17: 1663-1676.

Kuhl, M., Geis, K., Sheldahl, L.C., Pukrop, T., Moon, R.T., and Wedlich, D. 2001. Antagonistic regulation of convergent extension movements in Xenopus by Wnt/ $\beta$-catenin and Wnt/ $\mathrm{Ca}^{2+}$ signaling. Mech. Dev. 106: 61-76.

Larkin, K. and Danilchik, M.V. 1999. Ventral cell rearrangements contribute to anterior-posterior axis lengthening between neurula and tailbud stages in Xenopus laevis. Dev. Biol. 216: $550-560$.

Leaf, I., Tennessen, J., Mukhopadhyay, M., Westphal, H., and Shawlot, W. 2006. Sfrp5 is not essential for axis formation in the mouse. Genesis 44: 573-578.

Leimeister, C., Bach, A., and Gessler, M. 1998. Developmental expression patterns of mouse sFRP genes encoding members of the secreted frizzled related protein family. Mech. Dev. 75: $29-42$.

Leyns, L., Bouwmeester, T., Kim, S.H., Piccolo, S., and De Robertis, E.M. 1997. Frzb-1 is a secreted antagonist of Wnt signaling expressed in the Spemann organizer. Cell 88: 747756.

Liao, G., Tao, Q., Kofron, M., Chen, J.S., Schloemer, A., Davis, R.J., Hsieh, J.C., Wylie, C., Heasman, J., and Kuan, C.Y. 2006. Jun NH2-terminal kinase (JNK) prevents nuclear $\beta$ catenin accumulation and regulates axis formation in Xenopus embryos. Proc. Natl. Acad. Sci. 103: 16313-16318.

Macdonald, B.T., Semenov, M.V., and He, X. 2007. SnapShot: Wnt $/ \beta$-catenin signaling. Cell 131: 1204.

Mao, B., Wu, W., Li, Y., Hoppe, D., Stannek, P., Glinka, A., and Niehrs, C. 2001. LDL-receptor-related protein 6 is a receptor for Dickkopf proteins. Nature 411: 321-325.

Matsui, T., Raya, A., Kawakami, Y., Callol-Massot, C., Capdevila, J., Rodriguez-Esteban, C., and Izpisua Belmonte, J.C. 2005. Noncanonical Wnt signaling regulates midline convergence of organ primordia during zebrafish development. Genes \& Dev. 19: 164-175.

McLin, V.A., Rankin, S.A., and Zorn, A.M. 2007. Repression of $\mathrm{Wnt} / \beta$-catenin signaling in the anterior endoderm is essential for liver and pancreas development. Development 134: 2207-2217.

Mikels, A.J. and Nusse, R. 2006. Purified Wnt5a protein activates or inhibits $\beta$-catenin-TCF signaling depending on receptor context. PLoS Biol. 4: e115. doi: 10.1371/journal. pbio.0040115.

Moore-Scott, B.A., Opoka, R., Lin, S.C., Kordich, J.J., and Wells, J.M. 2007. Identification of molecular markers that are expressed in discrete anterior-posterior domains of the endoderm from the gastrula stage to mid-gestation. Dev. Dyn. 236: 1997-2003.

Mukhopadhyay, M., Shtrom, S., Rodriguez-Esteban, C., Chen, L., Tsukui, T., Gomer, L., Dorward, D.W., Glinka, A., Grinberg, A., Huang, S.P., et al. 2001. Dickkopf1 is required for embryonic head induction and limb morphogenesis in the mouse. Dev. Cell 1: 423-434.

Nojima, M., Suzuki, H., Toyota, M., Watanabe, Y., Maruyama, R., Sasaki, S., Sasaki, Y., Mita, H., Nishikawa, N., Yamaguchi, K., et al. 2007. Frequent epigenetic inactivation of SFRP genes and constitutive activation of Wnt signaling in gastric cancer. Oncogene 26: 4699-4713. 
Nomoto, S., Kinoshita, T., Kato, K., Otani, S., Kasuya, H., Takeda, S., Kanazumi, N., Sugimoto, H., and Nakao, A. 2007. Hypermethylation of multiple genes as clonal markers in multicentric hepatocellular carcinoma. Br. I. Cancer 97: $1260-1265$.

Ogata, S., Morokuma, J., Hayata, T., Kolle, G., Niehrs, C., Ueno, N., and Cho, K.W. 2007. TGF- $\beta$ signaling-mediated morphogenesis: Modulation of cell adhesion via cadherin endocytosis. Genes \& Dev. 21: 1817-1831.

Oishi, I., Suzuki, H., Onishi, N., Takada, R., Kani, S., Ohkawara, B., Koshida, I., Suzuki, K., Yamada, G., Schwabe, G.C., et al. 2003. The receptor tyrosine kinase Ror2 is involved in noncanonical Wnt5a/JNK signalling pathway. Genes Cells 8: 645-654.

Pandur, P., Lasche, M., Eisenberg, L.M., and Kuhl, M. 2002. Wnt-11 activation of a non-canonical Wnt signalling pathway is required for cardiogenesis. Nature 418: 636-641.

Park, M. and Moon, R.T. 2002. The planar cell-polarity gene stbm regulates cell behaviour and cell fate in vertebrate embryos. Nat. Cell Biol. 4: 20-25.

Park, T.J., Gray, R.S., Sato, A., Habas, R., and Wallingford, J.B. 2005. Subcellular localization and signaling properties of dishevelled in developing vertebrate embryos. Curr. Biol. 15: 1039-1044.

Pilcher, K.E. and Krieg, P.A. 2002. Expression of the Wnt inhibitor, sFRP5, in the gut endoderm of Xenopus. Gene Expr. Patterns 2: 369-372.

Rothbächer, U., Laurent, M.N., Deardorff, M.A., Klein, P.S., Cho, K.W., and Fraser, S.E. 2000. Dishevelled phosphorylation, subcellular localization and multimerization regulate its role in early embryogenesis. EMBO J. 19: 1010-1022.

Satoh, W., Gotoh, T., Tsunematsu, Y., Aizawa, S., and Shimono, A. 2006. Sfrp1 and Sfrp2 regulate anteroposterior axis elongation and somite segmentation during mouse embryogenesis. Development 133: 989-999.

Satoh, W., Matsuyama, M., Takemura, H., Aizawa, S., and Shimono, A. 2008. Sfrp1, Sfrp2, and Sfrp5 regulate the Wnt/ $\beta$ catenin and the planar cell polarity pathways during early trunk formation in mouse. Genesis 46: 92-103.

Schambony, A. and Wedlich, D. 2007. Wnt-5A/Ror2 regulate expression of XPAPC through an alternative noncanonical signaling pathway. Dev. Cell 12: 779-792.

Semenov, M.V., Tamai, K., Brott, B.K., Kuhl, M., Sokol, S., and He, X. 2001. Head inducer Dickkopf-1 is a ligand for Wnt coreceptor LRP6. Curr. Biol. 11: 951-961.

Semenov, M.V., Habas, R., Macdonald, B.T., and He, X. 2007. SnapShot: Noncanonical Wnt signaling pathways. Cell 131: 1378.

Sheldahl, L.C., Slusarski, D.C., Pandur, P., Miller, J.R., Kuhl, M., and Moon, R.T. 2003. Dishevelled activates $\mathrm{Ca}^{2+}$ flux, PKC, and CamKII in vertebrate embryos. J. Cell Biol. 161: 769-777.

Shook, D.R., Majer, C., and Keller, R. 2004. Pattern and morphogenesis of presumptive superficial mesoderm in two closely related species, Xenopus laevis and Xenopus tropicalis. Dev. Biol. 270: 163-185.

Sinner, D., Rankin, S., Lee, M., and Zorn, A.M. 2004. Sox17 and $\beta$-catenin cooperate to regulate the transcription of endodermal genes. Development 131: 3069-3080.

Sokol, S.Y., Klingensmith, J., Perrimon, N., and Itoh, K. 1995. Dorsalizing and neuralizing properties of Xdsh, a maternally expressed Xenopus homolog of dishevelled. Development 121: $1637-1647$.

Tada, M. and Smith, J.C. 2000. Xwnt11 is a target of Xenopus Brachyury: Regulation of gastrulation movements via Di- shevelled, but not through the canonical Wnt pathway. Development 127: 2227-2238.

Tahinci, E. and Symes, K. 2003. Distinct functions of Rho and Rac are required for convergent extension during Xenopus gastrulation. Dev. Biol. 259: 318-335.

Tao, Q., Yokota, C., Puck, H., Kofron, M., Birsoy, B., Yan, D., Asashima, M., Wylie, C.C., Lin, X., and Heasman, J. 2005. Maternal wnt11 activates the canonical wnt signaling pathway required for axis formation in Xenopus embryos. Cell 120: $857-871$.

Topol, L., Jiang, X., Choi, H., Garrett-Beal, L., Carolan, P.J., and Yang, Y. 2003. Wnt-5a inhibits the canonical Wnt pathway by promoting GSK-3-independent $\beta$-catenin degradation. $J$. Cell Biol. 162: 899-908.

Tzahor, E. and Lassar, A.B. 2001. Wnt signals from the neural tube block ectopic cardiogenesis. Genes \& Dev. 15: 255-260.

Ulrich, F., Krieg, M., Schotz, E.M., Link, V., Castanon, I., Schnabel, V., Taubenberger, A., Mueller, D., Puech, P.H., and Heisenberg, C.P. 2005. Wnt11 functions in gastrulation by controlling cell cohesion through Rab5c and E-cadherin. Dev. Cell 9: 555-564.

Wallingford, J.B. and Habas, R. 2005. The developmental biology of Dishevelled: An enigmatic protein governing cell fate and cell polarity. Development 132: 4421-4436.

Wallingford, J.B., Rowning, B.A., Vogeli, K.M., Rothbacher, U., Fraser, S.E., and Harland, R.M. 2000. Dishevelled controls cell polarity during Xenopus gastrulation. Nature 405: 8185.

Wang, S., Krinks, M., and Moos Jr., M. 1997. Frzb-1, an antagonist of Wnt-1 and Wnt- 8 , does not block signaling by Wnts3A, -5A, or -11. Biochem. Biophys. Res. Commun. 236: 502504.

Wang, J., Hamblet, N.S., Mark, S., Dickinson, M.E., Brinkman, B.C., Segil, N., Fraser, S.E., Chen, P., Wallingford, J.B., and Wynshaw-Boris, A. 2006. Dishevelled genes mediate a conserved mammalian PCP pathway to regulate convergent extension during neurulation. Development 133: 1767-1778.

Wawrzak, D., Metioui, M., Willems, E., Hendrickx, M., de Genst, E., and Leyns, L. 2007. Wnt3a binds to several sFRPs in the nanomolar range. Biochem. Biophys. Res. Commun. 357: 1119-1123.

Winklbauer, R., Medina, A., Swain, R.K., and Steinbeisser, H. 2001. Frizzled-7 signalling controls tissue separation during Xenopus gastrulation. Nature 413: 856-860.

Witzel, S., Zimyanin, V., Carreira-Barbosa, F., Tada, M., and Heisenberg, C.P. 2006. Wnt11 controls cell contact persistence by local accumulation of Frizzled 7 at the plasma membrane. J. Cell Biol. 175: 791-802.

Yamanaka, H., Moriguchi, T., Masuyama, N., Kusakabe, M., Hanafusa, H., Takada, R., Takada, S., and Nishida, E. 2002. JNK functions in the non-canonical Wnt pathway to regulate convergent extension movements in vertebrates. EMBO Rep. 3: 69-75.

Zaret, K.S. 2002. Regulatory phases of early liver development: Paradigms of organogenesis. Nat. Rev. Genet. 3: 499-512.

Zeng, X., Huang, H., Tamai, K., Zhang, X., Harada, Y., Yokota, C., Almeida, K., Wang, J., Doble, B., Woodgett, J., et al. 2008. Initiation of Wnt signaling: Control of Wnt coreceptor Lrp6 phosphorylation/activation via frizzled, dishevelled and axin functions. Development 135: 367-375.

Zou, H., Molina, J.R., Harrington, J.J., Osborn, N.K., Klatt, K.K., Romero, Y., Burgart, L.J., and Ahlquist, D.A. 2005. Aberrant methylation of secreted frizzled-related protein genes in esophageal adenocarcinoma and Barrett's esophagus. Int. J. Cancer 116: 584-591. 


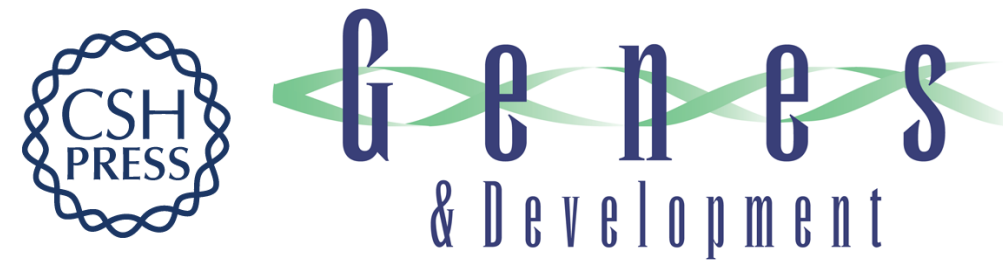

\section{Sfrp5 coordinates foregut specification and morphogenesis by antagonizing both canonical and noncanonical Wnt11 signaling}

Yan Li, Scott A. Rankin, Débora Sinner, et al.

Genes Dev. 2008, 22:

Access the most recent version at doi:10.1101/gad.1687308

Supplemental http://genesdev.cshlp.org/content/suppl/2008/11/06/22.21.3050.DC1
Material

References This article cites 73 articles, 25 of which can be accessed free at:

http://genesdev.cshlp.org/content/22/21/3050.full.html\#ref-list-1

License

Email Alerting Receive free email alerts when new articles cite this article - sign up in the box at the top

Service

right corner of the article or click here.

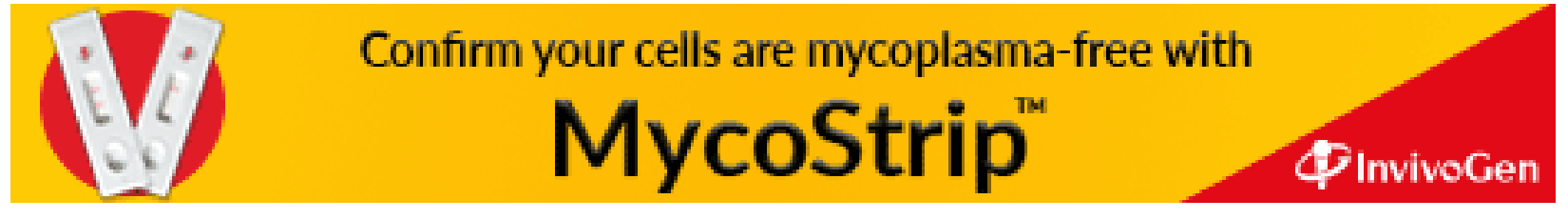

